\title{
Invariance results for definable extensions of groups
}

\author{
Mário J. Edmundo* \\ CMAF Universidade de Lisboa \\ Av. Prof. Gama Pinto 2 \\ 1649-003 Lisboa, Portugal
}

\author{
Gareth O. Jones ${ }^{\dagger}$ \\ School of Mathematics \\ University of Manchester \\ Oxford Road, Manchester, M13 9PL, UK
}

\author{
Nicholas J. Peatfield \\ 5 Dunkerry Road \\ Windmill Hill \\ Bristol \\ BS3 4LD, UK
}

June 10, 2010

\begin{abstract}
We show that in an o-minimal expansion of an ordered group finite definable extensions of a definable group which is defined in a reduct are already defined in the reduct. A similar result is proved for finite topological extensions of definable groups defined in o-minimal expansions of the ordered set of real numbers.
\end{abstract}

${ }^{*}$ With partial support from the FCT (Fundação para a Ciência e Tecnologia) program POCI 2010 (Portugal/FEDER-EU) and FCT (Fundação para a Ciência e Tecnologia) project PTDC/MAT/101740/2008.

${ }^{\dagger}$ Supported by NSERC while a post-doc at McMaster University, Canada. MSC: 03C64; 20E99. Keywords and phrases: O-minimal structures, definable groups. 


\section{Introduction}

In this paper we work in an arbitrary o-minimal structure $\mathcal{N}$ and by definable we mean definable in $\mathcal{N}$ (possibly) with parameters. We will assume that the reader is familiar with the basic theory of o-minimality ([1]), definable groups (e.g. [2], [4], [8], [17], [18], [19], [20] and [21]) and of locally definable groups ([3], [5], [6] and [17]). When working in the category of locally definable groups we will assume that $\mathcal{N}$ is $\aleph_{1}$-saturated.

The main theorem of the paper is the following:

Theorem 1.1 Let $\mathcal{K}$ be an o-minimal structure definable in $\mathcal{N}$ and let $G$ be $a \mathcal{K}$-definably connected locally $\mathcal{K}$-definable group defined without parameters and $p_{H}: H \rightarrow G$ a (locally) definable covering homomorphism. If there exists an admissible cover $\mathcal{U}=\left\{U_{\alpha}\right\}_{\alpha \in I}$ of $G$ by open $\mathcal{K}$-definable subsets defined without parameters over which $p_{H}: H \rightarrow G$ is trivial, then $p_{H}$ : $H \rightarrow G$ is (locally) definably isomorphic to a (locally) $\mathcal{K}$-definable covering homomorphism defined without parameters.

When $\mathcal{N}$ is an o-minimal expansion of an ordered group we have:

Corollary 1.2 Let $\mathcal{K}$ an o-minimal expansion of an ordered group definable in $\mathcal{N}$ and let $G$ be a $\mathcal{K}$-definably connected locally $\mathcal{K}$-definable group defined without parameters and $p_{H}: H \rightarrow G$ a (locally) definable covering homomorphism. Then $p_{H}: H \rightarrow G$ is (locally) definably isomorphic to a (locally) $\mathcal{K}$-definable covering homomorphism defined without parameters.

The next result is another consequence of the main theorem which was first observed in an unpublished manuscript by the authors and was quoted in the paper [13], where a different proof is presented, in connection with Pillay's conjectures ([22]). The fundamental results of the theory definable groups, which includes real algebraic groups and semi-algebraic groups, hinted at a deep connection between definable groups and Lie groups, which was finally formulated in the paper [22] as Pillay's conjecture for definable groups and the compact domination conjecture. Pillay's conjecture is a nonstandard analogue of Hilbert's $5^{\circ}$ problem for locally compact topological groups. On the other hand, the compact domination conjecture formalizes the idea that the quotient map in Pillay's conjecture should be a kind of intrinsic "standard part map". These conjectures lead to the development of new model theoretic tools as well as new geometric tools in o-minimality ([7], [8], [9], [10], [12], [13], [14], [15], [16]).

Corollary 1.3 Suppose that $\mathcal{N}$ is an o-minimal expansion of an ordered group. If $H$ is a definably connected, semi-simple definable group, then there exists a definable real closed field $R$ such that $H$ is definably isomorphic in $\mathcal{N}$, over parameters, to an $R$-semi-algebraic group $H^{\prime}$ defined without parameters. 
The technique used to prove the main theorem also gives with minor adaptations the following:

Theorem 1.4 Let $\mathcal{K}$ an $\aleph_{1}$-saturated elementary extension of an o-minimal expansion of the ordered set of real numbers. Let $G$ be a $\mathcal{K}$-definably connected locally $\mathcal{K}$-definable group defined without parameters and $p_{H}: H \rightarrow$ $G(\mathbb{R})$ a topological covering homomorphism with countable discrete kernel. Then there are a locally $\mathcal{K}$-definable group $H^{\prime}$ and a locally $\mathcal{K}$-definable covering homomorphism $p_{H^{\prime}}: H^{\prime} \rightarrow G$ all defined without parameters such that $H^{\prime}(\mathbb{R})=H$ and $p_{H}: H \rightarrow G(\mathbb{R})$ is topologically isomorphic to $p_{H^{\prime}(\mathbb{R})}$ : $H^{\prime}(\mathbb{R}) \rightarrow G(\mathbb{R})$. Moreover, if $G$ is $\mathcal{K}$-definable and the kernel of $p_{H}: H \rightarrow$ $G(\mathbb{R})$ is finite, then $p_{H^{\prime}}: H^{\prime} \rightarrow G$ is $\mathcal{K}$-definable defined without parameters.

\section{On (locally) definable covering maps}

Here we introduce some terminology and prove some preliminary results that will be useful later. A locally definable manifold (of dimension $n$ ) is a triple $\left(S,\left(U_{i}, \theta_{i}\right)_{i \leq \kappa}\right)$ with $\kappa \leq \omega$ where:

- $S=\bigcup_{i \leq \kappa} U_{i}$

- each $\theta_{i}: U_{i} \rightarrow N^{n}$ is an injection such that $\theta_{i}\left(U_{i}\right)$ is an open definable subset of $N^{n}$;

- for all $i, j, \theta_{i}\left(U_{i} \cap U_{j}\right)$ is an open definable subset of $\theta_{i}\left(U_{i}\right)$ and the transition maps $\theta_{i j}: \theta_{i}\left(U_{i} \cap U_{j}\right) \rightarrow \theta_{j}\left(U_{i} \cap U_{j}\right): x \mapsto \theta_{j}\left(\theta_{i}^{-1}(x)\right)$ are definable homeomorphisms.

We call the $\left(U_{i}, \theta_{i}\right)$ 's the definable charts of $S$. If $\kappa<\omega$ then $S$ is a definable manifold.

A (locally) definable manifold $S$ has a topology such that each $U_{i}$ is open and the $\theta_{i}$ 's are homeomorphisms: a subset $U$ of $S$ is an open in the basis for this topology if and only if for each $i, \theta_{i}\left(U \cap U_{i}\right)$ is an open definable subset of $\theta_{i}\left(U_{i}\right)$.

We say that a subset $A$ of $S$ is definable if and only if there is a finite $I_{0} \subseteq \kappa$ such that $A \subseteq \bigcup_{i \in I_{0}} U_{i}$ and for each $i \in I_{0}, \theta_{i}\left(A \cap U_{i}\right)$ is a definable subset of $\theta_{i}\left(U_{i}\right)$, and, a subset $B$ of $S$ is locally definable if and only if for each $i, B \cap U_{i}$ is a definable subset of $S$. We say that a (locally) definable manifold $S$ is definably connected if it is not the disjoint union of two open and closed locally definable subsets.

Let $\mathcal{U}=\left\{U_{\alpha}\right\}_{\alpha \in I}$ be a cover of $S$ by open definable subsets. We say that $\mathcal{U}$ is admissible if for each $i \leq \kappa$, the cover $\left\{U_{\alpha} \cap U_{i}\right\}_{\alpha \in I}$ of $U_{i}$ admits a finite subcover. If $\mathcal{V}=\left\{V_{\beta}\right\}_{\beta \in J}$ is another cover of $S$ by open definable subsets, we say that $\mathcal{V}$ refines $\mathcal{U}$, denoted $\mathcal{V} \leq \mathcal{U}$, if there is a map $\epsilon: J \rightarrow I$ such that $V_{\beta} \subseteq U_{\epsilon(\beta)}$ for all $\beta \in J$. 
A map $f: X \longrightarrow Y$ between locally definable manifolds with definable charts $\left(U_{i}, \theta_{i}\right)_{i \leq \kappa_{1}}$ and $\left(V_{j}, \delta_{j}\right)_{j \leq \kappa_{2}}$ respectively is a locally definable map if for every finite $I \subseteq \kappa_{1}$ there is a finite $J \subseteq \kappa_{2}$ such that:

- $f\left(\bigcup_{i \in I} U_{i}\right) \subseteq \bigcup_{j \in J} V_{j}$;

- the restriction $f_{\mid}: \bigcup_{i \in I} U_{i} \longrightarrow \bigcup_{j \in J} V_{j}$ is a definable map between definable manifolds, i.e., for each $i \in I$ and every $j \in J$ with $f\left(U_{i}\right) \cap$ $V_{j} \neq \emptyset, \delta_{j} \circ f \circ \theta_{i}^{-1}: \theta_{i}\left(U_{i}\right) \longrightarrow \delta_{j}\left(V_{j}\right)$ is a definable map between definable sets.

Thus we have the category of (locally) definable manifolds with (locally) definable continuous maps.

Given definably connected locally definable manifold $S$, a locally definable manifold $X$ and an admissible cover $\mathcal{U}=\left\{U_{\alpha}\right\}_{\alpha \in I}$ of $S$ by open definable subsets, we say that a continuous surjective locally definable map $p_{X}: X \rightarrow S$ is a locally definable covering map trivial over $\mathcal{U}=\left\{U_{\alpha}\right\}_{\alpha \in I}$ if the following holds:

- $p_{X}^{-1}\left(U_{\alpha}\right)=\dot{\bigcup}_{i \leq \lambda} U_{\alpha}^{i}$ (a disjoint union) of open definable subsets of $X$ with $\lambda \leq \omega$;

- each $p_{X \mid U_{\alpha}^{i}}: U_{\alpha}^{i} \rightarrow U_{\alpha}$ is a definable homeomorphism,

If both $S$ and $X$ are definable manifolds we say that $p_{X}: X \rightarrow S$ is a definable covering map trivial over $\mathcal{U}=\left\{U_{\alpha}\right\}_{\alpha \in I}$. A (locally) definable covering map $p_{X}: X \rightarrow G$ is a (locally) definable covering map trivial over some admissible cover $\mathcal{U}=\left\{U_{\alpha}\right\}_{\alpha \in I}$ of $S$ by open definable subsets.

If $Z$ is a countable discrete group, we say that a (locally) definable covering map $p_{X}: X \rightarrow S$ trivial over $\mathcal{U}=\left\{U_{\alpha}\right\}_{\alpha \in I}$ is a (locally) definable $Z$-covering map trivial over $\mathcal{U}=\left\{U_{\alpha}\right\}_{\alpha \in I}$ if in addition there exists a continuous (locally) definable action $Z \times X \rightarrow X:(z, x) \mapsto x^{z}$ of $Z$ on $X$ such that the following hold:

- For every $x \in S$ we have an induced action of $Z$ on $p_{X}^{-1}(x)=\left\{x_{i}: i \leq\right.$ $|Z|\}$ such that for any $i, j$ there is a unique $z \in Z$ such that $x_{j}=x_{i}^{z}$.

- For every $\alpha \in I$ we have an induced action of $Z$ on $p_{X}^{-1}\left(U_{\alpha}\right)=\left\{U_{\alpha}^{i}\right.$ : $i \leq|Z|\}$ such that for any $i, j$ there is a unique $z \in Z$ such that $U_{\alpha}^{j}=\left(U_{\alpha}^{i}\right)^{z}$.

(Note that when each $U_{\alpha}$ is definably connected, the first condition above implies the second). A (locally) definable $Z$-covering map $p_{X}: X \rightarrow G$ is a (locally) definable $Z$-covering map trivial over some admissible cover $\mathcal{U}=\left\{U_{\alpha}\right\}_{\alpha \in I}$ of $S$ by open definable subsets.

We say that two (locally) definable $Z$-covering maps $p_{X}: X \rightarrow S$ and $p_{Y}: Y \rightarrow S$ (trivial over $\mathcal{U}$ ) are (locally) definably homeomorphic if there is a (locally) definable homeomorphism $F: X \rightarrow Y$ such that: 
- $p_{X}=p_{Y} \circ F$.

- For every $x \in X$ and $z \in Z$, we have $F\left(x^{z}\right)=F(x)^{z}$.

The first observation of the paper is:

Lemma 2.1 Let $S$ be a definably connected locally definable manifold of dimension $n, \mathcal{U}=\left\{U_{\alpha}\right\}_{\alpha \in I}$ an admissible cover of $S$ by open definably connected definable subsets which refines the collection of definable charts of $S$ and $p_{X}: X \rightarrow S$ a surjective map.

(1) If for each $\alpha \in I$ we have

- $p_{X}^{-1}\left(U_{\alpha}\right)=\dot{\bigcup}_{i \leq \lambda} U_{\alpha}^{i}$ (a disjoint union) of subsets of $X$ with $\lambda \leq \omega$;

- each $p_{X \mid U_{\alpha}^{i}}: U_{\alpha}^{i} \rightarrow U_{\alpha}$ is a bijection;

then there exists a locally definable manifold structure on $X$ of dimension $n$ such that $p_{X}: X \rightarrow S$ is a locally definable covering map trivial $\operatorname{over} \mathcal{U}=\left\{U_{\alpha}\right\}_{\alpha \in I}$.

(2) If $Z$ is a countable discrete group and in addition there is an action $Z \times X \rightarrow X:(z, x) \mapsto x^{z}$ of $Z$ on $X$ such that, for each $\alpha \in I$ the following two conditions hold

- for every $x \in S$ we have an induced action of $Z$ on $p_{X}^{-1}(x)=\left\{x_{i}\right.$ : $i \leq|Z|\}$ such that for any $i, j$ there is a unique $z \in Z$ such that $x_{j}=x_{i}^{z} ;$ and

- there is an induced action of $Z$ on $p_{X}^{-1}\left(U_{\alpha}\right)=\left\{U_{\alpha}^{i}: i \leq|Z|\right\}$ such that for any $i, j$ there is a unique $z \in Z$ such that $U_{\alpha}^{j}=\left(U_{\alpha}^{i}\right)^{z}$;

then $p_{X}: X \rightarrow S$ is a locally definable $Z$-covering map trivial over $\mathcal{U}=\left\{U_{\alpha}\right\}_{\alpha \in I}$.

Proof. Let $\left(U_{i}, \theta_{i}\right)_{i \leq \kappa}$ be the definable charts of $S$ and let $\iota: I \rightarrow \kappa$ be a map such that $U_{\alpha} \subseteq U_{\iota(\alpha)}$ for all $\alpha \in I$. Let $\theta_{\alpha}:=\theta_{\iota(\alpha) \mid U_{\alpha}}$. Since $\mathcal{U}=\left\{U_{\alpha}\right\}_{\alpha \in I}$ is an admissible cover of $S$ by open definable subsets $\mathcal{U}$ has a countable subcover. Thus, without loss of generality, we may assume that $I$ is countable.

For each $\alpha \in I$ and $i \leq \lambda$, set $\delta_{\alpha, i}: U_{\alpha}^{i} \rightarrow \theta_{\alpha}\left(U_{\alpha}\right): x \mapsto \theta_{\alpha} \circ p_{X \mid U_{\alpha}^{i}}$. We claim that the $\left(U_{\alpha}^{i}, \delta_{\alpha, i}\right)$ 's are the charts making $X$ into a locally definable manifold of dimension $n$. Clearly we have:

- $X=\cup\left\{U_{\alpha}^{i}: \alpha \in I, i \leq \lambda\right\}$

- each $\delta_{\alpha, i}: U_{\alpha}^{i} \rightarrow N^{n}$ is an injection such that $\delta_{\alpha, i}\left(U_{\alpha}^{i}\right)=\theta_{\alpha}\left(U_{\alpha}\right)$ is an open definable subset of $N^{n}$; 
- for all $\alpha, i, \beta, j, \delta_{\alpha, i}\left(U_{\alpha}^{i} \cap U_{\beta}^{j}\right)=\theta_{\alpha}\left(U_{\alpha} \cap U_{\beta}\right)$ is an open definable subset of $\delta_{\alpha, i}\left(U_{\alpha}^{i}\right)=\theta_{\alpha}\left(U_{\alpha}\right)$ and the transition maps $\delta_{(\alpha, i),(\beta, j)}: \delta_{\alpha, i}\left(U_{\alpha}^{i} \cap\right.$ $\left.U_{\beta}^{j}\right) \rightarrow \delta_{\beta, j}\left(U_{\alpha}^{i} \cap U_{\beta}^{j}\right): x \mapsto \delta_{\beta, j}\left(\lambda_{\alpha, i}^{-1}(x)\right)=\theta_{\beta}\left(\theta_{\alpha}^{-1}(x)\right)$ are definable homeomorphisms.

When $X$ is equipped with this locally definable manifold structure, the map $p_{X}: X \rightarrow S$ is a continuous locally definable map since $\theta_{\alpha} \circ p_{X \mid U_{\alpha}^{i}} \circ \delta_{\alpha, i}^{-1}$ : $\delta_{\alpha, i}\left(U_{\alpha}^{i}\right) \rightarrow \theta_{\alpha}\left(U_{\alpha}\right)$ is the identity map on $\theta_{\alpha}\left(U_{\alpha}\right)$. Furthermore, by the hypothesis on $p_{X}: X \rightarrow S$, this map is a locally definable covering map trivial over $\mathcal{U}=\left\{U_{\alpha}\right\}_{\alpha \in I}$.

(2) Since $Z$ is a countable discrete group, to show that the action $Z \times$ $X \rightarrow X:(z, x) \mapsto x^{z}$ of $Z$ on $X$ is continuous and locally definable it is enough to show that for each $z \in Z$, the map $X \rightarrow X: x \mapsto x^{z}$ is continuous and locally definable. But through the charts, this map is $\delta_{\alpha, i}\left(U_{\alpha}^{i}\right) \rightarrow \delta_{\alpha, j}\left(U_{\alpha}^{j}\right): x \mapsto \delta_{\alpha, j}\left(\left(\delta_{\alpha, i}^{-1}(x)\right)^{z}\right)$, where $j$ is the unique element such that $U_{\alpha}^{j}=\left(U_{\alpha}^{i}\right)^{z}$, and therefore it is the identity map on $\theta_{\alpha}\left(U_{\alpha}\right)$. Thus $p_{X}: X \rightarrow S$ is a locally definable $Z$-covering map trivial over $\mathcal{U}=\left\{U_{\alpha}\right\}_{\alpha \in I}$.

An immediate consequence of Lemma 2.1 is:

Corollary 2.2 Let $\mathcal{K}$ be an o-minimal structure definable in $\mathcal{N}$. Let $S$ be a $\mathcal{K}$-definably connected locally $\mathcal{K}$-definable manifold defined without parameters and $p_{X}: X \rightarrow S$ a (locally) definable covering (resp. Z-covering) map. Suppose that there exists an admissible cover $\mathcal{V}=\left\{V_{\alpha}\right\}_{\alpha \in I}$ of $S$ by $\mathcal{K}$-definable open subsets defined without parameters such that:

- $p_{X}: X \rightarrow S$ is trivial over $\mathcal{V}=\left\{V_{\alpha}\right\}_{\alpha \in I}$.

- $\mathcal{V}=\left\{V_{\alpha}\right\}_{\alpha \in I}$ refines the collection of definable charts of $S$.

Then $p_{X}: X \rightarrow S$ is (locally) definably homeomorphic to a (locally) $\mathcal{K}$ definable covering (resp. Z-covering) map trivial over $\mathcal{V}=\left\{V_{\alpha}\right\}_{\alpha \in I}$.

Similarly we have:

Corollary 2.3 Let $\mathcal{K}$ be an o-minimal expansion of the ordered set of real numbers. Let $S$ be a $\mathcal{K}$-definably connected locally $\mathcal{K}$-definable manifold defined without parameters and $p_{X}: X \rightarrow S$ a topological covering (resp. $Z$-covering) map with countable fibers. Suppose that there exists an admissible cover $\mathcal{V}=\left\{V_{\alpha}\right\}_{\alpha \in I}$ of $S$ by $\mathcal{K}$-definable open subsets defined without parameters such that:

- $p_{X}: X \rightarrow S$ is trivial over $\mathcal{V}=\left\{V_{\alpha}\right\}_{\alpha \in I}$.

- $\mathcal{V}=\left\{V_{\alpha}\right\}_{\alpha \in I}$ refines the collection of definable charts of $S$. 
Then $p_{X}: X \rightarrow S$ is topologically homeomorphic to a locally $\mathcal{K}$-definable covering (resp. Z-covering) map trivial over $\mathcal{V}=\left\{V_{\alpha}\right\}_{\alpha \in I}$. Moreover, if $S$ is $\mathcal{K}$-definable and the fibers of $p_{X}: X \rightarrow S$ are finite, then $p_{X}: X \rightarrow S$ is topologically homeomorphic to a $\mathcal{K}$-definable covering (resp. $Z$-covering) map trivial over $\mathcal{V}=\left\{V_{\alpha}\right\}_{\alpha \in I}$

The following lemma will also be quite useful later. In recent work by Edmundo, Eleftheriou and Prelli the assumptions of this lemma are proved. Note however, that in this paper we apply the lemma for locally definable groups in which case the assumptions were verified in [6].

Lemma 2.4 Let $\mathcal{K}$ be an o-minimal expansion of an ordered group (resp. of the ordered set of real numbers). Let $S$ be a $\mathcal{K}$-definably connected locally $\mathcal{K}$-definable manifold and suppose that there exists an admissible cover $\mathcal{U}=\left\{U_{\alpha}\right\}_{\alpha \in I}$ of $S$ by $\mathcal{K}$-definable open subsets of $S$ such that each $U_{\alpha}$ is $\mathcal{K}$-definably connected and $\mathcal{K}$-definably simply connected (resp. connected and simply connected). Then every locally $\mathcal{K}$-definable covering map (resp. topological covering map) $p_{X}: X \rightarrow S$ is trivial over $\mathcal{U}=\left\{U_{\alpha}\right\}_{\alpha \in I}$.

Proof. Let $p_{X}: X \rightarrow S$ be a locally $\mathcal{K}$-definable covering map (resp. a topological covering map). For each $\alpha \in I$ set $p_{\mid X}^{-1}\left(U_{\alpha}\right)=\dot{\bigcup}_{i \leq \lambda_{\alpha}} U_{\alpha}^{i}$ with $\lambda_{\alpha} \leq \omega$ where the $U_{\alpha}^{i}$ 's are the $\mathcal{K}$-definably connected components (resp. connected components) of $p_{\mid X}^{-1}\left(U_{\alpha}\right)$. We have to show that:

- each $p_{\mid X}: U_{\alpha}^{i} \rightarrow U_{\alpha}$ is a $\mathcal{K}$-definable (resp. topological) homeomorphism;

- $\lambda_{\alpha}=\lambda_{\beta}$ for all $\alpha, \beta \in I$.

Note that the first point implies that $\lambda_{\alpha}=\left|p_{X}^{-1}(x)\right|$ for all $x \in U_{\alpha}$. In particular, if $U_{\alpha} \cap U_{\beta} \neq \emptyset$ then $\lambda_{\alpha}=\lambda_{\beta}$. Since $S$ is $\mathcal{K}$-definably connected, we must have also $\lambda_{\alpha}=\lambda_{\beta}$ for all $\alpha, \beta \in I$ as required. On the other hand, since $p_{X}: X \rightarrow S$ is a locally $\mathcal{K}$-definable covering map (resp. a topological covering map) it is by definition a local homeomorphism. So it is enough to show that each $p_{X \mid U_{\alpha}^{i}}: U_{\alpha}^{i} \rightarrow U_{\alpha}$ is a bijection.

We show first that $p_{X \mid U_{\alpha}^{i}}: U_{\alpha}^{i} \rightarrow U_{\alpha}$ is surjective. Let $u \in U_{\alpha}^{i}$ and $x=p_{X \mid U_{\alpha}^{i}}(u) \in U_{\alpha}$. Let $y \in U_{\alpha}$ and since $U_{\alpha}$ is $\mathcal{K}$-definably path connected (resp. path connected), let $\gamma$ be a $\mathcal{K}$-definable path (resp. path) in $U_{\alpha}$ from $x$ to $y$. By [6] Lemma 2.7 (1) (resp. [11] Chapter 11, Proposition 11.6), there is a unique $\mathcal{K}$-definable path (resp. path) $\widetilde{\gamma}$ in $X$, lifting $\gamma$, and starting at $u$. The image of $\widetilde{\gamma}$ is a $\mathcal{K}$-definably connected (resp. connected) subset of $\dot{U}_{i \leq \lambda_{\alpha}} U_{\alpha}^{i}$ and intersects $U_{\alpha}^{i}$. Thus this image is contained in $U_{\alpha}^{i}$ and the endpoint $v$ of $\widetilde{\gamma}$ is also contained in $U_{\alpha}^{i}$ and is mapped by $p_{X \mid U_{\alpha}^{i}}$ to $y$.

Suppose that $u, v \in U_{\alpha}^{i}$ are such that $p_{X \mid U_{\alpha}^{i}}(u)=x=p_{X \mid U_{\alpha}^{i}}(v)$. Since $U_{\alpha}^{i}$ is $\mathcal{K}$-definably connected (resp. connected), it is $\mathcal{K}$-definably path connected 
by [1] Chapter 6, Proposition 3.2 (resp. path connected because it also locally path connected as $p_{X}$ is a local homeomorphism and $U_{\alpha}$ is locally path connected). Let $\gamma$ be a $\mathcal{K}$-definable path (resp. path) in $U_{\alpha}^{i}$ from $u$ to $v$. Then $\sigma=p_{X \mid U_{\alpha}^{i}} \circ \gamma$ is a $\mathcal{K}$-definable loop (resp. loop) in $U_{\alpha}$ at $x$ and $\gamma$ is the unique $\mathcal{K}$-definable lifting (resp. lifting) of $\sigma$ to $U_{\alpha}^{i}$ starting at $u$. Since the o-minimal fundamental groups (resp. the topological fundamental groups) $\pi_{1}\left(U_{\alpha}\right)$ are trivial, it follows from [6] Remark 2.9 (1) (resp. [11] Chapter 13, Exercise 13.2) that $u=v$. So $p_{X \mid U_{\alpha}^{i}}: U_{\alpha}^{i} \rightarrow U_{\alpha}$ is injective.

\section{On definable covering homomorphisms}

Recall that a group $(G, \cdot)$ is a locally definable group over $A$, with $A \subseteq N$ and $|A|<\aleph_{1}$, if there is a countable collection $\left\{Z_{i}: i \in I\right\}$ of definable subsets of $N^{n}$, all definable over $A$, such that:

- $G=\cup\left\{Z_{i}: i \in I\right\}$;

- for every $i, j \in I$ there is $k \in I$ such that $Z_{i} \cup Z_{j} \subseteq Z_{k}$;

- the restriction of the group multiplication to $Z_{i} \times Z_{j}$ is a definable map over $A$ into $N^{n}$.

Given two locally definable groups $H$ and $G$ over $A$, we say that $H$ is a locally definable subgroup of $G$ over $A$ if $H$ is a subgroup of $G$. A homomorphism $\alpha: G \longrightarrow H$ between locally definable groups over $A$ is called a locally definable homomorphism over $A$ if for every definable subset $Z \subseteq G$ defined over $A$, the restriction $\alpha_{\mid Z}$ is a definable map over $A$.

In the terminology of [17], locally definable groups (respectively homomorphisms) are $\bigvee$-definable groups (respectively homomorphisms). Therefore, every locally definable group $G \subseteq N^{n}$ over $A$ is equipped with a unique topology $\tau$, called the $\tau$-topology, such that: (i) $(G, \tau)$ is a topological group; (ii) every generic element of $G$ has an open definable neighborhood $U \subseteq N^{n}$ such that $U \cap G$ is $\tau$-open and the topology which $U \cap G$ inherits from $\tau$ agrees with the topology it inherits from $N^{n}$; (iii) locally definable homomorphisms between locally definable groups are continuous with respect to the $\tau$ topologies. Note also that when $G$ is a definable group, then its $\tau$ topology coincides with the its t-topology from [21]. In fact, just like in the definable case $([21])$ we have the following (see the proof of Proposition 3.3 in $[6])$ :

Proposition 3.1 ([6]) Let $G$ be a locally definable group of dimension $k$ and defined without parameters. Then there is a countable collection $\left\{O_{s}\right.$ : $s \in S\}$ of $\tau$-open definably $\tau$-connected definable subsets of $G$ defined without 
parameters and a countable collection $\left\{\theta_{s}: O_{s} \rightarrow N^{k}: s \in S\right\}$ of definable homeomorphisms defined without parameters such that:

- $G=\cup\left\{O_{s}: s \in S\right\}$;

- each $\theta_{s}: O_{s} \rightarrow N^{k}$ is a definable homeomorphism onto an open cell in $N^{k}$

- for all $s, t, \theta_{s}\left(O_{s} \cap O_{t}\right)$ is an open definable subset of $\theta_{s}\left(O_{s}\right)$ and the transition maps $\theta_{s t}: \theta_{s}\left(O_{s} \cap O_{t}\right) \rightarrow \theta_{t}\left(O_{s} \cap O_{t}\right): x \mapsto \theta_{t}\left(\theta_{s}^{-1}(x)\right)$ are definable homeomorphisms.

In particular, $\left(O_{s}, \theta_{s}\right)_{s \in S}$ is a locally definable manifold structure on $G$.

We say that a locally definable group $G$ is definably connected if it so as a locally definable manifold. This is equivalent to notion of definably $\tau$-connected from [5] and it corrigendum.

Another result that will be useful below is the following (see the proof of Theorem 3.6 in [3]):

Theorem $3.2([3])$ Let $p_{H}: H \longrightarrow G$ be a surjective locally definable homomophism over $A$ between locally definable groups over $A$. If $\operatorname{Ker}_{H}$ has dimension zero, then $p_{H}: H \longrightarrow G$ is a locally definable covering homomorphism over $A$. In fact, $p_{H}: H \longrightarrow G$ is a locally definable $\operatorname{Ker} p_{H}$-covering homomorphism over $A$.

So we often call a surjective (locally) definable homomorphism $p_{H}$ : $H \rightarrow G$ with a (necessarily (locally) definable) kernel $Z$ of dimension zero a (locally) definable $Z$-covering homomorphism (trivial over some admissible cover $\mathcal{U}=\left\{U_{\alpha}\right\}_{\alpha \in I}$ of $G$ by open definable subsets). We say that two (locally) definable $Z$-covering homomorphisms $p_{H}: H \rightarrow G$ and $p_{H^{\prime}}: H^{\prime} \rightarrow$ $G$ (trivial over some admissible cover $\mathcal{U}=\left\{U_{\alpha}\right\}_{\alpha \in I}$ of $G$ by open definable subsets) are (locally) definably isomorphic if there is a (locally) definable isomorphism $F: H \rightarrow H^{\prime}$ such that:

- $p_{H}=p_{H^{\prime}} \circ F$.

- For every $x \in X$ and $z \in Z$, we have $F\left(x^{z}\right)=F(x)^{z}$.

We are now ready to present the proof of Theorem 1.1. The argument of this proof applies with obvious modifications also to the proof of the following Theorem which is needed in the proof of Theorem 1.4. Thus we will run the two proofs in parallel. 
Theorem 3.3 Let $\mathcal{K}$ be an $\aleph_{1}$-saturated elementary extension of an o-minimal expansion of the ordered set of real numbers. Let $G$ be a $\mathcal{K}$-definably connected locally $\mathcal{K}$-definable group defined without parameters and $p_{H}: H \rightarrow$ $G(\mathbb{R})$ a topological covering homomorphism with countable discrete kernel. Suppose that there exists an admissible cover $\mathcal{U}=\left\{U_{\alpha}\right\}_{\alpha \in I}$ of $G$ by open $\mathcal{K}$-definable subsets defined without parameters such that $p_{H}: H \rightarrow G(\mathbb{R})$ is trivial over $\mathcal{U}(\mathbb{R})=\left\{U_{\alpha}(\mathbb{R})\right\}_{\alpha \in I}$. Then there are a locally $\mathcal{K}$-definable group $H^{\prime}$ and a locally $\mathcal{K}$-definable covering homomorphism $p_{H^{\prime}}: H^{\prime} \rightarrow G$ all defined without parameters such that $H^{\prime}(\mathbb{R})=H$ and $p_{H}: H \rightarrow G(\mathbb{R})$ is topologically isomorphic to $p_{H^{\prime}(\mathbb{R})}: H^{\prime}(\mathbb{R}) \rightarrow G(\mathbb{R})$. Moreover, if $G$ is $\mathcal{K}$-definable and the kernel of $p_{H}: H \rightarrow G(\mathbb{R})$ is finite, then $p_{H^{\prime}}: H^{\prime} \rightarrow G$ is $\mathcal{K}$-definable defined without parameters.

Proof of Theorem 1.1 (resp. Theorem 3.3): Let $\mathcal{K}, G$ and $p_{H}$ : $H \rightarrow G\left(\right.$ resp. $\left.p_{H}: H \rightarrow G(\mathbb{R})\right)$ be as in the assumptions of Theorem 1.1 (resp. Theorem 3.3). Assume also that there exists an admissible cover $\mathcal{U}=\left\{U_{\alpha}\right\}_{\alpha \in I}$ of $G$ by open $\mathcal{K}$-definable subsets defined without parameters such that $p_{H}: H \rightarrow G$ (resp. $\left.p_{H}: H \rightarrow G(\mathbb{R})\right)$ is trivial over $\mathcal{U}=\left\{U_{\alpha}\right\}_{\alpha \in I}$ (resp. $\mathcal{U}(\mathbb{R})=\left\{U_{\alpha}(\mathbb{R})\right\}_{\alpha \in I}$ ). Let $\mathcal{K}(\mathbb{R})$ is the o-minimal expansion of the ordered set of real number of which $\mathcal{K}$ of Theorem 3.3 is an $\aleph_{1}$-saturated elementary extension.

By taking a refinement we may assume without loss of generality that the admissible cover $\mathcal{U}=\left\{U_{\alpha}\right\}_{\alpha \in I}$ of $G$ refines the collection of definable charts of $G$. By Corollary 2.2 (resp. Corollary 2.3) we may assume that $p_{H}: H \rightarrow G$ (resp. $p_{H}: H \rightarrow G(\mathbb{R})$ ) is a locally $\mathcal{K}$-definable covering map defined without parameters trivial over $\mathcal{U}=\left\{U_{\alpha}\right\}_{\alpha \in I}$ (resp. $\mathcal{U}(\mathbb{R})=$ $\left\{U_{\alpha}(\mathbb{R})\right\}_{\alpha \in I}$ ). Thus it remains to show that the group operation on $H$ is locally $\mathcal{K}$-definable and defined without parameters.

To simplify the notation below we will still write $\mathcal{K}$ for $\mathcal{K}(\mathbb{R}), G$ for $G(\mathbb{R})$ and $\mathcal{U}=\left\{U_{\alpha}\right\}_{\alpha \in I}$ for $\mathcal{U}(\mathbb{R})=\left\{U_{\alpha}(\mathbb{R})\right\}_{\alpha \in I}$.

We have the following commutative diagram

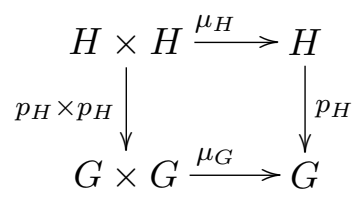

where $\mu_{H}$ (resp. $\mu_{G}$ ) is the group operation in $H$ (resp. in $G$ ). For $\alpha \in I$, let $p_{H}^{-1}\left(U_{\alpha}\right)=\dot{\bigcup}_{i \leq \lambda} U_{\alpha}^{i}$. Since the $U_{\alpha}^{i}$ 's are $\mathcal{K}$-definable and defined without parameters, to reach our goal it is enough to show that each restriction $\mu_{H \mid U_{\alpha}^{i} \times U_{\beta}^{j}}$ is $\mathcal{K}$-definable and defined without parameters.

Fix $\alpha, \beta \in I$ and $i, j \leq \lambda$. For $\gamma \in I$ and $k \leq \lambda$ consider the subsets

$$
A_{\alpha, \beta ; \gamma}^{i, j ; k}=\left\{(x, y) \in U_{\alpha}^{i} \times U_{\beta}^{j} \mid \mu_{H}(x, y) \in U_{\gamma}^{k}\right\}
$$


and

$$
A_{\alpha, \beta ; \gamma}^{i, j}=\left\{(x, y) \in U_{\alpha}^{i} \times U_{\beta}^{j} \mid \mu_{H}(x, y) \in p_{H}^{-1}\left(U_{\gamma}\right)\right\}
$$

of $U_{\alpha}^{i} \times U_{\beta}^{j}$. Consider also the $\mathcal{K}$-definable subset

$$
A_{\alpha, \beta ; \gamma}=\left\{(u, v) \in U_{\alpha} \times U_{\beta} \mid \mu_{G}(u, v) \in U_{\gamma}\right\}
$$

of $U_{\alpha} \times U_{\beta}$ defined without parameters. By the commutativity of the previous diagram we clearly we have

$$
\left(p_{H \mid U_{\alpha}^{i}}\right)^{-1} \times\left(p_{H \mid U_{\beta}^{j}}\right)^{-1}\left(A_{\alpha, \beta ; \gamma}\right)=A_{\alpha, \beta ; \gamma}^{i, j}
$$

and so $A_{\alpha, \beta ; \gamma}^{i, j}$ is $\mathcal{K}$-definable and defined without parameters. Clearly

$$
A_{\alpha, \beta ; \gamma}^{i, j}=\bigcup_{k \leq \lambda} A_{\alpha, \beta ; \gamma}^{i, j ; k}
$$

and by the continuity of $\mu_{H \mid A_{\alpha, \beta ; \gamma}^{i, j}}: A_{\alpha, \beta ; \gamma}^{i, j} \rightarrow p_{H}^{-1}\left(U_{\gamma}\right)$, each $A_{\alpha, \beta ; \gamma}^{i, j ; k}$ is clopen in $A_{\alpha, \beta ; \gamma}^{i, j}$.

Claim 3.4 Each $A_{\alpha, \beta ; \gamma}^{i, j ; k}$ is $\mathcal{K}$-definable and defined without parameters and

$$
A_{\alpha, \beta ; \gamma}^{i, j}=A_{\alpha, \beta ; \gamma}^{i, j ; k_{1}} \dot{\cup} \cdots \dot{\cup} A_{\alpha, \beta ; \gamma}^{i, j ; k_{\gamma}}
$$

for some finite $k_{\gamma}$.

Proof. Take a cell decomposition $\mathcal{C}$ of $A_{\alpha, \beta ; \gamma}^{i, j}$ in the prime model of $\mathcal{K}$ over the empty set. Since each cell is $\mathcal{N}$-definably connected (resp. is connected) and each $A_{\alpha, \beta ; \gamma}^{i, j ; k}$ which is non empty is $\mathcal{N}$-definable and clopen (resp. clopen), each cell in $\mathcal{C}$ is contained in an $\mathcal{N}$-definably connected component (resp. connected component) of some $A_{\alpha, \beta ; \gamma}^{i, j ; k}$. Therefore, each non empty $A_{\alpha, \beta ; \gamma}^{i, j ; k}$ is a finite union of cells in $\mathcal{C}$ and therefore it is $\mathcal{K}$-definable and defined without parameters. On the other hand, since $A_{\alpha, \beta ; \gamma}^{i, j}$ has finitely many $\mathcal{K}$-definably connected components and each $A_{\alpha, \beta ; \gamma}^{i, j ; k}$ which is non empty is clopen and $\mathcal{K}$-definable we must have $A_{\alpha, \beta ; \gamma}^{i, j}=A_{\alpha, \beta ; \gamma}^{i, j ; k_{1}} \dot{\cup} \cdots \dot{\cup} A_{\alpha, \beta ; \gamma}^{i, j ; k_{\gamma}}$ for some finite $k_{\gamma}$.

Since for each $k \in\left\{k_{1}, \ldots, k_{\gamma}\right\}$ we have

$$
\mu_{H \mid A_{\alpha, \beta ; \gamma}^{i, j ; k}}=\left(p_{H \mid U_{\gamma}^{k}}\right)^{-1} \circ \mu_{G} \circ\left(p_{H \mid U_{\alpha}^{i}} \times p_{H \mid U_{\beta}^{j}}\right)
$$

it follows from Claim 3.4 that each such $\mu_{H \mid A_{\alpha, \beta ; \gamma}^{i, j ; k}}$ is $\mathcal{K}$-definable and defined without parameters. 
Claim 3.5 There is a finite subset $J_{\alpha, \beta}^{i, j} \subseteq I$ such that

$$
U_{\alpha}^{i} \times U_{\beta}^{j}=\bigcup\left\{A_{\alpha, \beta ; \gamma}^{i, j ; k} \mid \gamma \in J_{\alpha, \beta}^{i, j}, k \in\left\{k_{1}, \ldots, k_{\gamma}\right\}\right\} .
$$

Proof. Since $\mu_{G}\left(U_{\alpha} \times U_{\beta}\right)$ is a $\mathcal{K}$-definable subset of $G$ defined without parameters and $\mathcal{U}=\left\{U_{\alpha}\right\}_{\alpha \in I}$ is an admissible cover of $G$ by open $\mathcal{K}$-definable subsets defined without parameters, there is a finite subset $J_{\alpha, \beta}^{i, j} \subseteq I$ such that $\mu_{G}\left(U_{\alpha} \times U_{\beta}\right) \subseteq \bigcup\left\{U_{\gamma} \mid \gamma \in J_{\alpha, \beta}^{i, j}\right\}$. Since $p_{H} \circ \mu_{H}=\mu_{G} \circ\left(p_{H} \times p_{H}\right)$ we have $\mu_{H}\left(U_{\alpha}^{i} \times U_{\beta}^{j}\right) \subseteq \bigcup\left\{p_{H}^{-1}\left(U_{\gamma}\right) \mid \gamma \in J_{\alpha, \beta}^{i, j}\right\}$ and the result follows from this by the definition of the $A_{\alpha, \beta ; \gamma}^{i, j ; k}$ 's.

It follows from this Claim 3.5 and the fact that each $\mu_{H \mid A_{\alpha, \beta ; \gamma}^{i, j ; k}}$ with $k \in\left\{k_{1}, \ldots, k_{\gamma}\right\}$ is $\mathcal{K}$-definable and defined without parameters that each $\mu_{H \mid U_{\alpha}^{i} \times U_{\beta}^{j}}$ is $\mathcal{K}$-definable and defined without parameters as required concluding in this way the proof of Theorem 1.1 (resp. Theorem 3.3). Moreover, if $p_{H}: H \rightarrow G$ (resp. $p_{H}: H \rightarrow G(\mathbb{R})$ is definable (resp. has finite kernel and $G$ is $\mathcal{K}$-definable), then $p_{H}: H \rightarrow G$ is definably isomorphic to a $\mathcal{K}$-definable covering homomorphism defined without parameters (resp. $p_{H^{\prime}}: H^{\prime} \rightarrow G$ is $\mathcal{K}$-definable defined without parameters).

For the proof of Corollary 1.2 we require the following lemma:

Lemma 3.6 Let $\mathcal{K}$ be an o-minimal expansion of an ordered group and $G$ a $\mathcal{K}$-definably connected locally $\mathcal{K}$-definable group defined without parameters. Then there exists an admissible cover $\mathcal{U}=\left\{U_{\alpha}\right\}_{\alpha \in I}$ of $G$ by $\mathcal{K}$-definable open subsets of $G$ defined without parameters such that each $U_{\alpha}$ is $\mathcal{K}$-definably connected and $\mathcal{K}$-definably simply connected. In particular, by Lemma 2.4, every locally $\mathcal{K}$-definable covering map $p_{X}: X \rightarrow G$ is trivial over $\mathcal{U}=$ $\left\{U_{\alpha}\right\}_{\alpha \in I}$.

On the other hand for the proof of Theorem 1.4 we require the following analogue of Lemma 3.6:

Lemma 3.7 Let $\mathcal{K}$ be an $\aleph_{1}$-saturated elementary extension of an o-minimal expansion of the ordered set of real numbers. Let $G$ a $\mathcal{K}$-definably connected locally $\mathcal{K}$-definable group defined without parameters. Then there exists an admissible cover $\mathcal{U}=\left\{U_{\alpha}\right\}_{\alpha \in I}$ of $G$ by $\mathcal{K}$-definable open subsets of $G$ defined without parameters such that each $U_{\alpha}(\mathbb{R})$ is connected and simply connected. In particular, by Lemma 2.4, every topological covering map $p_{X}: X \rightarrow G(\mathbb{R})$ is trivial over $\mathcal{U}(\mathbb{R})=\left\{U_{\alpha}(\mathbb{R})\right\}_{\alpha \in I}$.

As before the proofs of Lemma 3.6 and Lemma 3.7 will run in parallel. 
Proof of Lemma 3.6 (resp. Lemma 3.7): Let $\mathcal{K}$ and $G$ be as in the assumptions of Lemma 3.6 (resp. Lemma 3.7). Let $\mathcal{K}(\mathbb{R})$ is the o-minimal expansion of the ordered set of real number of which $\mathcal{K}$ of Lemma 3.7 is an $\aleph_{1}$-saturated elementary extension.

By Theorem 3.1, let $\mathcal{U}=\left\{U_{\alpha}\right\}_{\alpha \in I}$ be an admissible cover of $G$ by $\mathcal{K}$ definably connected, open $\mathcal{K}$-definable subsets of $G$ defined without parameters each of which is $\mathcal{K}$-definably homeomorphic over the empty set to an open $\mathcal{K}$-cell in $K^{n}$ where $n=\operatorname{dim} G$. By [1] Chapter 6, Proposition 3.2, the $U_{\alpha}$ 's are $\mathcal{K}$-definably connected, $\mathcal{K}$-definably path connected and by [6] Proposition 3.3, the o-minimal fundamental groups $\pi_{1}\left(U_{\alpha}\right)$ are trivial (resp. by similar arguments, the $U_{\alpha}(\mathbb{R})$ 's are connected, path connected and the topological fundamental groups $\pi_{1}\left(U_{\alpha}(\mathbb{R})\right)$ are trivial).

Proof of Corollary 1.2: Let $\mathcal{K}$ an o-minimal expansion of an ordered group definable in $\mathcal{N}$ and let $G$ be a $\mathcal{K}$-definably connected locally $\mathcal{K}$-definable group defined without parameters and $p_{H}: H \rightarrow G$ a locally definable covering homomorphism.

By definition $p_{H}: H \rightarrow G$ is a locally definable covering map trivial over some admissible cover $\mathcal{W}=\left\{W_{\alpha}\right\}_{\alpha \in L}$ of $G$ by definable in $\mathcal{N}$ open subsets of $G$. Let $\mathcal{J}$ be the o-minimal expansion of $\mathcal{K}$ expanded by all the definable in $\mathcal{N}$ subsets of each $K^{m}$. In particular, the $W_{\alpha}$ 's are $\mathcal{J}$ definable open subsets of $G$ defined without parameters. By Theorem 1.1 applied to the definable o-minimal structure $\mathcal{J}$ in $\mathcal{N}, p_{H}: H \rightarrow G$ is locally definably isomorphic to a locally $\mathcal{J}$-definable covering homomorphism. By Lemmas 2.4 and 3.6 and Theorem 1.1 applied to $\mathcal{K}$ and $\mathcal{J}$ instead of $\mathcal{K}$ and $\mathcal{N}, p_{H}: H \rightarrow G$ is locally $\mathcal{J}$-definably isomorphic to a locally $\mathcal{K}$ definable covering homomorphism defined without parameters. Moreover, if $p_{H}: H \rightarrow G$ is definable, then $p_{H}: H \rightarrow G$ is definably isomorphic to a $\mathcal{K}$-definable covering homomorphism defined without parameters.

Proof of Corollary 1.3: Let $G$ be a definably connected, semi-simple definable group, i.e. it is non abelian and has no infinite normal abelian subgroups. In this case, the center $Z(G)$ of $G$ is finite and by [18] and [19] (the proof of Theorem 5.1 there), there is a definable real closed field $R$ and there are $R$-definably simple, $R$-definable groups $G_{i}$ defined without parameters such that $G / Z(G)$ is definably isomorphic to $G_{1} \times \cdots \times G_{l}$. Now apply Corollary 1.2 to the definable covering homomorphism $h: G \longrightarrow G_{1} \times$ $\cdots \times G_{l}$ which is the composition of the quotient definable homomorphism $G \longrightarrow G / Z(G)$ and the definable isomorphism $G / Z(G) \simeq G_{1} \times \cdots \times G_{l}$. 
Proof of Theorem 1.4: Let $\mathcal{K}$ an $\aleph_{1}$-saturated elementary extension of an o-minimal expansion of the ordered set of real numbers. Let $G$ be a $\mathcal{K}$ definably connected locally $\mathcal{K}$-definable group defined without parameters and $p_{H}: H \rightarrow G(\mathbb{R})$ a topological covering homomorphism with countable discrete kernel.

By Lemmas 2.4 and 3.7 and Theorem 3.3 there is a locally $\mathcal{K}$-definable covering homomorphism $p_{H^{\prime}}: H^{\prime} \rightarrow G$ defined without parameters such that $p_{H}: H \rightarrow G(\mathbb{R})$ is topologically isomorphic to $p_{H^{\prime}(\mathbb{R})}: H^{\prime}(\mathbb{R}) \rightarrow G(\mathbb{R})$. Moreover, if $G$ is $\mathcal{K}$-definable and the kernel of $p_{H}: H \rightarrow G(\mathbb{R})$ is finite, then $p_{H^{\prime}}: H^{\prime} \rightarrow G$ is $\mathcal{K}$-definable defined without parameters.

\section{References}

[1] L. van den Dries, Tame topology and o-minimal structures Cambridge University Press 1998.

[2] M. Edmundo Solvable groups definable in o-minimal structures J. Pure Appl. Algebra 185 (1-3) (2003) 103-145.

[3] M. Edmundo Covers of groups definable o-minimal structures Illinois J. Math. 49 (1) (2005) 99-120. Erratum Illinois J. Math. 51 (3) (2007) 1037-1038.

[4] M. Edmundo A remark on divisibility of definable groups Math. Logic Quart. 51 (6) (2005) 639-641.

[5] M. Edmundo Locally definable groups in o-minimal structures J. Algebra 301 (1) (2006) 194-223. Corrigendum (with E. Baro) J. Algebra 320 (7) (2008) 3079-3080.

[6] M. Edmundo and P. Eleftheriou The universal covering homomorphism in o-minimal expansions of groups Math. Log. Quart. 53 (6) (2007) 571-582.

[7] M. Edmundo, G. Jones and N. Peatfield Sheaf cohomology in o-minimal structures J. Math. Logic 6 (2) (2006) 163-179.

[8] M. Edmundo and M. Otero Definably compact abelian groups J. Math. Logic 4 (2) (2004) 163-180.

[9] P. Eleftheriou Groups definable in linear o-minimal structures Ph.D. Thesis, University of Notre Dame, 2007. 
[10] P. Eleftheriou and S. Starchenko Groups definable in ordered vector spaces over ordered division rings J. Symb. Logic 72 (2007) 1108-1140.

[11] W. Fulton Algebraic topology Springer Verlag 1995.

[12] E. Hrushovski, Y. Peterzil and A. Pillay Groups, measures and the NIP J. Amer. Math. Soc. 21 (2) (2008) 563-596.

[13] E. Hrushovski, Y. Peterzil, and A. Pillay On central extensions and definably compact groups in o-minimal structures Preprint Oct. 2008.

[14] E. Hrushovski and A. Pillay On NIP and invariant measures Preprint Jan. 2009.

[15] M. Otero and Y. Peterzil G-linear sets and torsion points in definably compact groups Archive Math. Logic (to appear).

[16] Y. Peterzil Returning to semi-bounded sets Preprint Oct. 2007.

[17] Y. Peterzil and S. Starchenko Definable homomorphisms of abelian groups definable in o-minimal structures Ann. Pure Appl. Logic 101 (1) (1999) 1-27.

[18] Y. Peterzil, A. Pillay and S. Starchenko Definably simple groups in o-minimal structures Trans. Amer. Math. Soc. 352 (10) (2000) 4397-4419.

[19] Y. Peterzil, A. Pillay, S. Starchenko Simple algebraic and semialgebraic groups over real closed fields Trans. Amer. Math. Soc. 352 (10) (2000) 4421-4450.

[20] Y. Peterzil, A. Pillay and S. Starchenko Linear groups definable in o-minimal structures J. Algebra 247 (2002) 1-23.

[21] A. Pillay On groups and fields definable in o-minimal structures J. Pure Appl. Algebra 53 (1988) 239-255.

[22] A. Pillay Type-definability, compact Lie groups and ominimality J. Math. Logic 4 (2) (2004) 147-162. 